\title{
Particle Physics with ORCA
}

\author{
Alba Domi $^{1,2,3, *}$, Simon Bourret ${ }^{4, * *}$, and Liam Quinn $^{3, * * *}$ on behalf of the KM3NeT Collab- \\ oration \\ ${ }^{1}$ Department of Physics, Universitá degli studi di Genova, Via Dodecaneso, 33, 16146 Genova \\ ${ }^{2}$ INFN, Genova, Via Dodecaneso, 33, 16146 Genova \\ ${ }^{3}$ Aix-Marseille Univ, CNRS/IN2P3, CPPM, Marseille, France \\ ${ }^{4}$ APC, Université Paris Diderot, CNRS/IN2P3, CEA/IRFU, Observatoire de Paris, Sorbonne Paris \\ Citeé, 75205 Paris, France
}

\begin{abstract}
KM3NeT is a Megaton-scale neutrino telescope currently under construction at the bottom of the Mediterranean Sea. When completed, it will consist of two separate detectors: ARCA (Astroparticle Research with Cosmics in the Abyss), optimised for high-energy neutrino astronomy, and ORCA (Oscillation Research with Cosmics in the Abyss) for neutrino oscillation studies of atmospheric neutrinos. The main goal of ORCA is the determination of the neutrino mass ordering (NMO). Nevertheless it is possible to exploit ORCA's configuration to make other important measurements, such as sterile neutrinos, non standard interactions, tau-neutrino appearance, neutrinos from Supernovae, Dark Matter and Earth Tomography studies. Part of these analyses are summarized here.
\end{abstract}

\section{Introduction}

Open issues in neutrino oscillations include the neutrino mass ordering, leptonic CP violation, and the $\theta_{23}$ octant. The neutrino mass ordering is a crucial missing piece in our understanding of neutrino mass. In order to measure it, the KM3NeT Collaboration is building a dense, deep-sea detector, ORCA (Oscillation Research with Cosmics in the Abyss), sensitive to atmospheric neutrino oscillations [1].

\section{The KM3NeT/ORCA Detector}

ORCA is located $40 \mathrm{~km}$ offshore Toulon (France), at a depth of $2450 \mathrm{~m}$, and about $10 \mathrm{~km}$ west of the site of the ANTARES neutrino telescope that is currenty operational [1]. Upon completion ORCA will consist of 120 flexible detection units (DUs), each of which comprises 18 Digital Optical Modules (DOMs). A DOM is a pressure resistant, 17" glass sphere containing a total of 31 3" Photomultiplier Tubes (PMTs) and their associated electronics. The vertical spacing between DOMs is $9 \mathrm{~m}$ and the DUs are located about $23 \mathrm{~m}$ from each other on the seafloor. Its geometry is optimised for particle physics studies with atmospheric neutrinos in the few $\mathrm{GeV}$ range. The total instrumented volume is approximately 8 Mton. The construction of the infrastructure has already started.

\footnotetext{
*e-mail: alba.domi@ge.infn.it

**e-mail: bourret@apc.in2p3.fr

***e-mail: quinn@cppm.in2p3.fr
} 


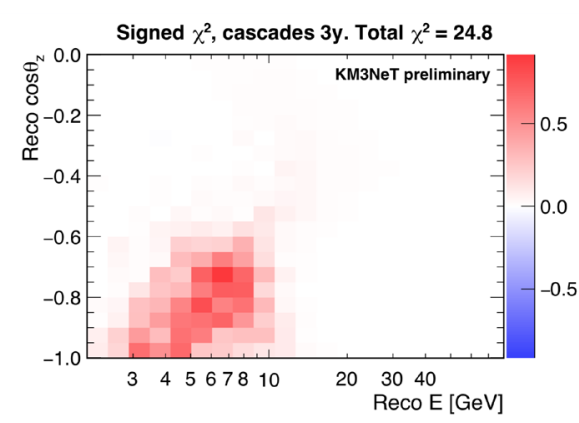

(a)

\begin{tabular}{|l|l|l|l|}
\hline Parameter & treatment & true value & prior \\
\hline$\left|\Delta \mathrm{m}_{31}^{2}\right|\left(\mathrm{eV}^{2}\right)$ & fitted & $2.4810^{-3}$ & free \\
$\Delta \mathrm{m}_{21}^{2}\left(\mathrm{eV}^{2}\right)$ & fix & $7.5310^{-5}$ & - \\
$\theta_{13}\left(^{\circ}\right)$ & fitted & 8.42 & 0.26 \\
$\theta_{12}\left({ }^{\circ}\right)$ & fix & 33.4 & - \\
$\theta_{23}\left({ }^{\circ}\right)$ & fitted & $38-52$ & free \\
$\delta_{C P}$ & fitted & $0,2 \pi$ & free \\
Flux spectral tilt & fitted & 0 & free \\
$v / \bar{v}$ skew & fitted & 0 & 0.03 \\
Tracks normalisation & fitted & 1 & free \\
Cascades normalisation & fitted & 1 & free \\
NC events normalisation & fitted & 1 & free \\
\hline
\end{tabular}

Figure 1. Signed $\chi^{2}$ shown as a function of reconstructed neutrino energy and co-sine zenith for shower-like events. Figure in Ref. [2]
Table 1. Oscillation and nuisance parameters, and systematic uncertainties used for the NMO study.

\section{Sensitivity to the Neutrino Mass Ordering}

In the flux of atmospheric neutrinos crossing the Earth, matter effects introduce a resonance into the oscillation probabilities with a maximum in the $\mathrm{GeV}$ range. The effect of the resonance is more pronounced in the shower channel (mostly $v_{e}$ ), as it is shown in Fig. 1 by the signed $\chi^{2}=\left(N_{N O}-N_{I O}\right)\left|N_{N O}-N_{I O}\right| / N_{N O}$, where $N_{N O}\left(N_{I O}\right)$ represents the expected number of shower-like events in case of NO (IO). Random decision forest algorithms are used to reject background (optical, atmospheric muons) and categorise events as either tracks (mostly $v_{\mu}$ ) and showers. Using this method, ORCA is able to achieve a partial statistical separation between $v_{\mu}$ and $v_{e}$ charged current events. The detector response is modelled by building a multi-dimensional response matrix from simulated events, incorporating the detection and selection efficiency, classification probabilities and correlated smearing of reconstructed energy and zenith angle. Fig. 2 shows the median significance to reject the wrong NMO hypothesis, based on the study of a log-likelihood ratio (LLR) test statistic for 3 years of data taking with the full ORCA detector and with the ensemble of nuisance parameters, oscillation and systematics shown in table 1 [2].

\section{Sterile Neutrinos and Non Standard Interactions}

With ORCA it is also possible to search for anomalies in the oscillation pattern arising from sterile neutrinos or non standard Interactions (NSI). In the sterile neutrino case, the Hamiltonian is extended to include four neutrinos. The fourth neutrino flavour is assumed not to interact with matter, yielding an effective potential difference between the sterile and active flavours due to neutral current interactions. In the NSI case, the interaction Hamiltonian of the neutrino system is modified into an arbitrary unitary form motivated by the potential existence of new neutrino interactions that behave as four-fermion point interactions at low energies. ORCA sensitivity to NSI parameters is shown in Ref. [3] and it improves on current limits by about an order of magnitude. An updated sensitivity to sterile neutrinos is shown in Fig. 3 (a); it shows the expected sensitivities of the ORCA detector to a sterile neutrino in the $\left|U_{\mu 4}\right|^{2}-\left|U_{\tau 4}\right|^{2}$ parameter space for 1 and 3 years of data taking assuming NO. Sensitivities were computed for $\Delta m_{41}^{2}=0.3 \mathrm{eV}^{2}$, but this result is expected to be valid for any $\Delta m_{41}^{2}>0.1 \mathrm{eV}^{2}$. 


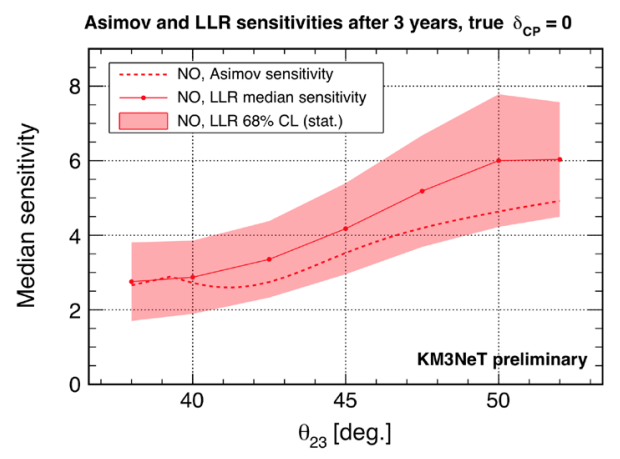

(a)

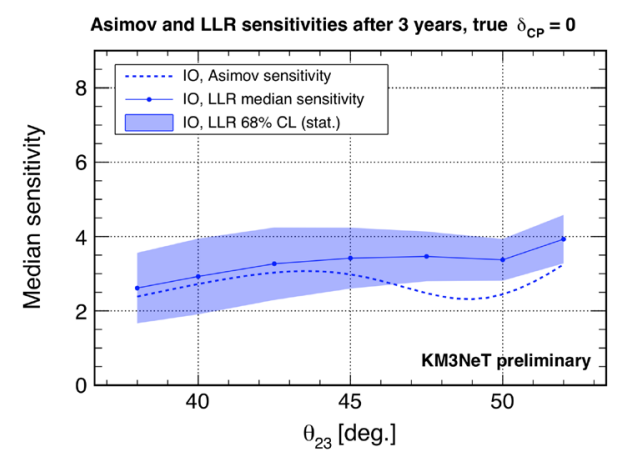

(b)

Figure 2. Sensitivity of ORCA to the NMO after 3 years of data taking: (a) true Normal Ordering (NO) and (b) true Inverted Ordering (IO). Figures and more details on the statistical methods in Ref. [2].

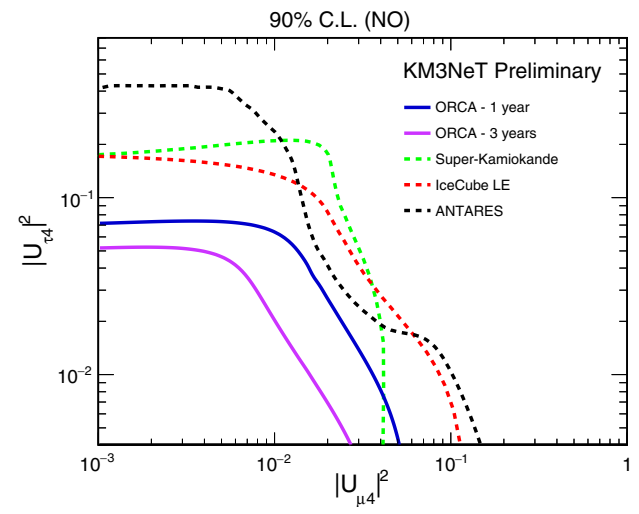

(a)

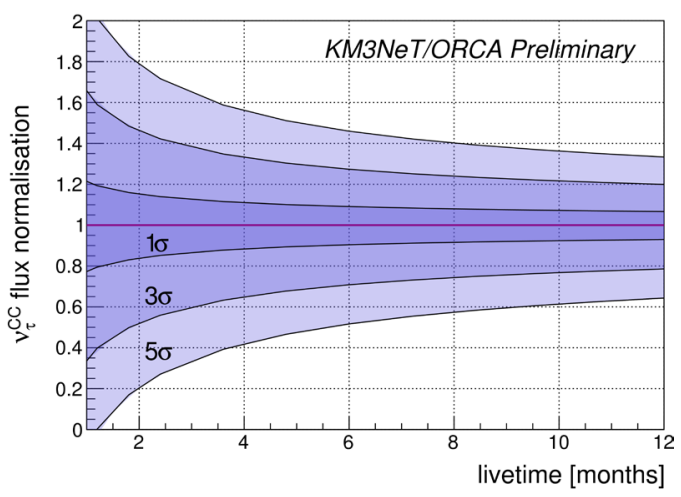

(b)

Figure 3. (a) ORCA sensitivity to a sterile neutrino for 1 and 3 years of data taking com-pared to upper limits from other experiments. (b) Sensitivity to $v_{\tau}$-flux normalisation for few months of full ORCA detector operation. Figure (b) in Ref. [5].

\section{Tau-neutrino Appearance}

Measurements of $v_{\tau}$ allow us to test the unitarity of the Pontecorvo-Maki-Nakagawa-Sakata (PMNS) matrix [4] and Beyond Standard Model (BSM) theories. Monte Carlo simulations predict more than $\sim 3,000 v_{\tau}$ events per year for the full ORCA detector. Most of them leave shower-like event signatures in the detector. Fig. 3 (b) shows the sensitivity to constrain the $v_{\tau}$ flux normalisation, provided the PMNS matrix is unitary, in few months of data taking with the full ORCA detector. It is possible to exclude non-appearance at the $5 \sigma$ level within 2 months of operation [5].

\section{Indirect Search of Dark Matter}

KM3NeT/ORCA also allows us to search for high energy neutrinos coming from Dark Matter self-annihilation in heavy celestial bodies, such as the Sun or the Galactic Centre, under the 


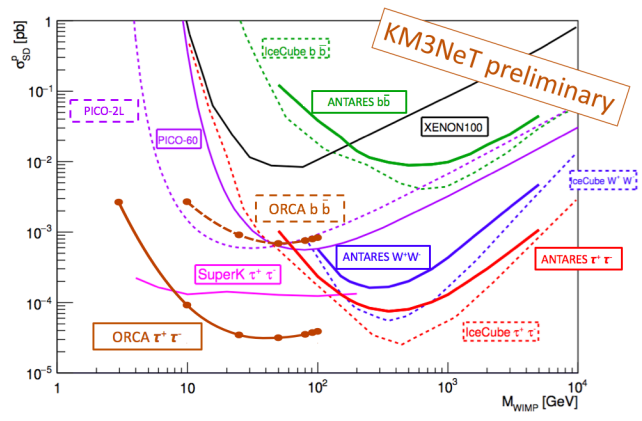

(a)

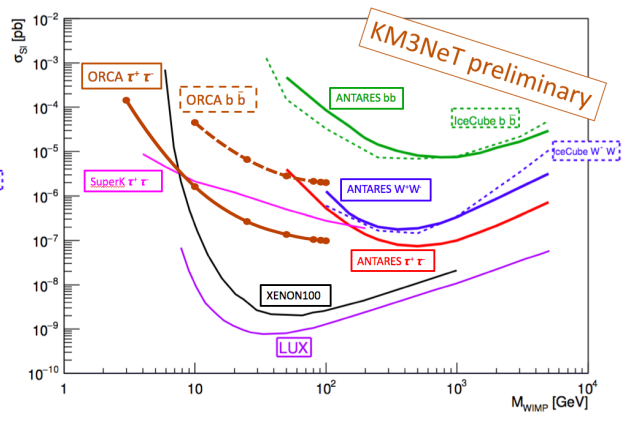

(b)

Figure 4. SD (a) and SI (b) cross section sensitiviy for ORCA for 3 years of data taking compared with the other Dark Matter searches. Figures in Ref. [6].

assumption that Dark Matter behaves like a Weakly Interacting Massive Particle (WIMP) that is captured by the gravitational field of a massive body. Consequently, a higher density of WIMPs is expected inside that body, leading to an increased annihilation rate into Standard Model particles, which can escape and decay, producing high energy neutrinos $\left(E_{v}>\mathrm{GeV}\right)$ that can be detected by a neutrino telescope. Since, for the case of the Sun or the Earth, the number of neutrinos observed from the source is related to the WIMP capture rate, this method enables us to constrain the WIMP-Nucleon cross section, which can be divided into a Spin Dependent (SD) and Spin Independent (SI) component. The sensitivity of ORCA to each these, for Dark Matter in the Sun, is shown in Fig. 4 for 3 years of data taking; in the case of SD cross sections, the ORCA sensitivity is very competitive with other Dark Matter searches.

\section{Conclusions}

KM3NeT/ORCA is an 8 Mton Cherenkov telescope under construction in the Mediterranean Sea optimised for particle physics studies with few-GeV atmospheric neutrinos. Within a few years of operation it will be able to measure the NMO with a sensitivity of $3 \sigma$ and it is competitive with other experiments to investigate neutrino properties and also to perform studies on a wide range of astroparticle physics. Details can be found in the KM3NeT Letter of Intent [1].

\section{References}

[1] The KM3NeT Collaboration, Letter of Intent, Journal of Physics G: Nuclear and Particle Physics, 43 (8), 084001, 2016

[2] S. Bourret and L. Quinn (KM3NeT Collab.), Neutrino2018, DOI: http://doi.org/10.5281/zenodo.1300771

[3] A. Kouchner, J. Coelho (KM3NeT Collab.), PoS (ICRC 2017), DOI: https://doi.org/10.22323/1.301.1027

[4] Maki, Z; Nakagawa, M.; Sakata, S. (1962). DOI: 10.1143/PTP.28.870.

[5] T. Eberl, S. Hallmann, J. Hofestädt and M. Moser (KM3NeT Collab.), Neutrino2018, DOI: http://doi.org/10.5281/zenodo.1292823

[6] V. Kulikovskiy (KM3NeT Collab.), ICRC 2017 (1142). 